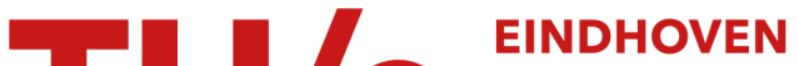

\section{Nonlinear driven response of a phase-field crystal in a periodic pinning potential}

\section{Citation for published version (APA):}

Achim, C. V., Ramos, J. A. P., Karttunen, M. E. J., Elder, K. R., Granato, E., Ala-Nissila, T., \& Ying, S-C. (2009). Nonlinear driven response of a phase-field crystal in a periodic pinning potential. Physical Review E - Statistical, Nonlinear, and Soft Matter Physics, 79(1), 011606-1/10. https://doi.org/10.1103/PhysRevE.79.011606

DOI:

10.1103/PhysRevE.79.011606

Document status and date:

Published: 01/01/2009

\section{Document Version:}

Publisher's PDF, also known as Version of Record (includes final page, issue and volume numbers)

\section{Please check the document version of this publication:}

- A submitted manuscript is the version of the article upon submission and before peer-review. There can be important differences between the submitted version and the official published version of record. People interested in the research are advised to contact the author for the final version of the publication, or visit the $\mathrm{DOI}$ to the publisher's website.

- The final author version and the galley proof are versions of the publication after peer review.

- The final published version features the final layout of the paper including the volume, issue and page numbers.

Link to publication

\section{General rights}

Copyright and moral rights for the publications made accessible in the public portal are retained by the authors and/or other copyright owners and it is a condition of accessing publications that users recognise and abide by the legal requirements associated with these rights.

- Users may download and print one copy of any publication from the public portal for the purpose of private study or research.

- You may not further distribute the material or use it for any profit-making activity or commercial gain

- You may freely distribute the URL identifying the publication in the public portal.

If the publication is distributed under the terms of Article 25fa of the Dutch Copyright Act, indicated by the "Taverne" license above, please follow below link for the End User Agreement:

www.tue.nl/taverne

Take down policy

If you believe that this document breaches copyright please contact us at:

openaccess@tue.nl

providing details and we will investigate your claim. 


\title{
Nonlinear driven response of a phase-field crystal in a periodic pinning potential
}

\author{
C. V. Achim, ${ }^{1}$ J. A. P. Ramos, ${ }^{2,3}$ M. Karttunen, ${ }^{4}$ K. R. Elder, ${ }^{5}$ E. Granato, ${ }^{3,6}$ T. Ala-Nissila, ${ }^{1,6}$ and S. C. Ying ${ }^{6}$ \\ ${ }^{1}$ Department of Applied Physics, Helsinki University of Technology, P.O. Box 1100, FIN-02015 TKK, Espoo, Finland \\ ${ }^{2}$ Departamento de Ciências Exatas, Universidade Estadual do Sudoeste da Bahia, 45000-000 Vitória da Conquista, BA, Brazil \\ ${ }^{3}$ Laboratório Associado de Sensores e Materiais, Instituto Nacional de Pesquisas Espaciais, São José dos Campos, São Paulo, Brazil \\ ${ }^{4}$ Department of Applied Mathematics, The University of Western Ontario, London (ON), Canada N6A 5B7 \\ ${ }^{5}$ Department of Physics, Oakland University, Rochester, Michigan 48309-4487, USA \\ ${ }^{6}$ Department of Physics, Brown University, Providence, Rhode Island 02912-1843, USA
}

(Received 29 September 2008; revised manuscript received 22 December 2008; published 26 January 2009)

\begin{abstract}
We study numerically the phase diagram and the response under a driving force of the phase field crystal model for pinned lattice systems introduced recently for both one- and two-dimensional systems. The model describes the lattice system as a continuous density field in the presence of a periodic pinning potential, allowing for both elastic and plastic deformations of the lattice. We first present results for phase diagrams of the model in the absence of a driving force. The nonlinear response to a driving force on an initially pinned commensurate phase is then studied via overdamped dynamic equations of motion for different values of mismatch and pinning strengths. For large pinning strength the driven depinning transitions are continuous, and the sliding velocity varies with the force from the threshold with power-law exponents in agreement with analytical predictions. Transverse depinning transitions in the moving state are also found in two dimensions. Surprisingly, for sufficiently weak pinning potential we find a discontinuous depinning transition with hysteresis even in one dimension under overdamped dynamics. We also characterize structural changes of the system in some detail close to the depinning transition.
\end{abstract}

DOI: 10.1103/PhysRevE.79.011606

PACS number(s): 68.43.De, 64.60.Cn, 05.40.-a

\section{INTRODUCTION}

There exist many systems in nature with two or more competing length scales, which often leads to the appearance of spatially modulated structures. Such systems may exhibit both commensurate $(C)$ and incommensurate $(I)$ phases $[1,2]$ characterized by differences in the spatial ordering of the system. Important examples include spin density waves $[3,4]$, charge density waves [5], vortex lattices in superconducting films with pinning centers [6], and weakly adsorbed monolayers $[7,8]$ on a substrate. The emerging structures are characterized by an order parameter (e.g, charge, spin or particle density) that is modulated in space with a given wave vector $q$. In particular, for two-dimensional (2D) adsorbate systems, there is competition between the commensurate state which is favored by a strong periodic pinning potential and the cost of the elastic energy depending on the mismatch between the intrinsic lattice constant $a$ of the overlayer, and the period $b$ of the pinning potential.

While the static properties of $C$ and $I$ structures have been extensively characterized $[1,2]$ much less is known about their dynamics. A particularly interesting case arises when an initially pinned phase is subjected to an external driving force $f$. The resulting nonlinear response is relevant for a variety of different physical systems which are accessible experimentally. A driven atomic monolayer in a periodic pinning potential is an interesting realization of such nonlinear behavior [9], which is directly relevant for experiments on sliding friction between two surfaces with a lubricant [10] and between adsorbed layers and an oscillating substrate $[11,12]$. Other systems of great interest are driven charge density waves [13-15] in which commensurability and impurity pinning often compete [16], and superconductor vor- tex arrays in which different commensurability and pinning behaviors have been experimentally observed [17-19], including periodic and asymmetric potentials.

For a sufficiently large pinning potential, the phase may remain pinned for small forces if there are no thermal fluctuations present. This means that at zero temperature there is a finite critical force $f_{c}$ above which the system starts moving. For many systems, it is found that just above the threshold $f_{c}$, the drift velocity $v_{d}$ shows a power-law dependence with respect to the force $f$

$$
v_{d} \propto\left(f-f_{c}\right)^{\zeta}
$$

When this behavior is regarded as a dynamical critical phenomenon, the power-law exponent $\zeta$ of the corresponding driven depinning transition can be argued to result from the scaling behavior of the system near the threshold [13] with corresponding divergent time and length scales and universal behavior. In general, however, the observed value of $\zeta$ may depend on the system and its dimensionality. For a pure elastic medium with quenched randomness there appears to be a universal value which depends on the dimensionality of the system, provided inertial effects are negligible [20,21]. For the case of an initially commensurate phase in a periodic pinning potential without disorder, a power-law exponent $\zeta$ $=1 / 2$ is expected independent of the dimension as the threshold behavior can be understood from the point of view of single particle behavior [13-15,22]. In the limit of a large force $\left(f-f_{c}\right) / f_{c} \gg 1$ the system is moving and the corresponding relationship between the driving force and the sliding velocity defines the sliding friction coefficient

$$
\eta_{s}=f / v_{d}
$$




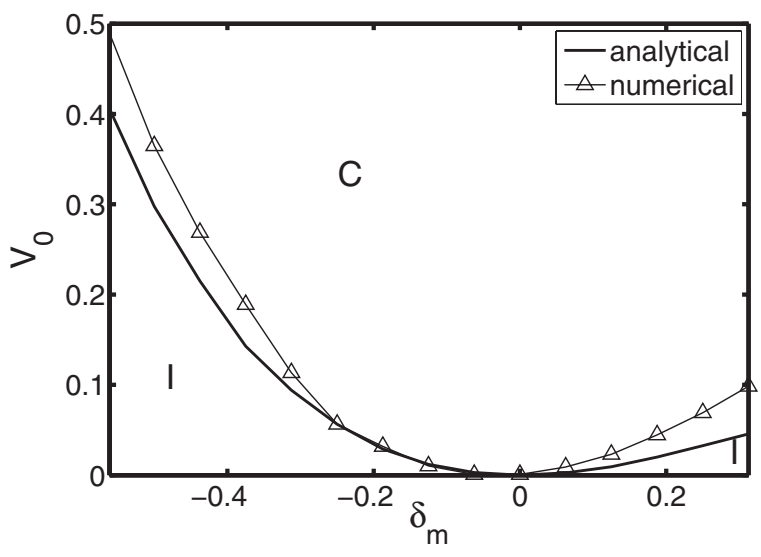

FIG. 1. Phase boundary between commensurate $(C)$ and incommensurate $(I)$ phases for the 1D pinned PFC model, calculated numerically (continuous line with triangles) and analytically (continuous line).

The simple Frenkel-Kontorova (FK) model [23,24] extended to two dimensions and other similar elastic models have been used to study driven depinning transitions and the sliding friction of adsorbed monolayers [9,25]. Although these models take into account topological defects in the form of domain walls they leave out plastic deformations of the layer due to other defects such as dislocations. These defects are particularly important when the CI transition occurs between two different crystal structures or in the presence of thermal fluctuations or quenched disorder, and should be taken into account for a more realistic description of the system. Such defects can be automatically included in a full microscopic model involving interacting atoms in the presence of a substrate potential using more realistic interaction potentials. However, the full complexities of the microscopic model severely limit the system sizes that can be studied numerically, even when simple Lennard-Jones potentials are used to describe the interactions [26,27].

When the driving depinning transition is discontinuous, hysteresis effects can occur which result in two different critical forces $f_{c}^{\text {in }}>f_{c}^{\text {de }}$, corresponding to the threshold values for increasing the force from zero and decreasing the force from a large value, respectively. A fundamental issue in modeling such systems is the origin of the hysteresis. It is well known that hysteresis can occur in underdamped systems, where inertia effects are present. However, molecular dynamics simulations of a 2D model of an adsorbed layer with Lennard-Jones interacting potential for increasing values of the damping coefficient (microscopic friction) and analytical arguments suggested that hysteresis should remain in the overdamped limit $[9,28]$. On the other hand, results for the pure elastic FK model shows that although the hysteresis behavior is similar to Lennard-Jones model for weak damping, it disappears in the overdamped limit [25]. The different behaviors could be due to the absence of some defects generated during the depinning transitions, which are allowed in the Lennard-Jones model but not in the elastic FK model. In fact, hysteresis can be argued to arise from topological defects in the lattice such as dislocations in two-dimensional systems [14] even in the absence of inertial effects. One thus
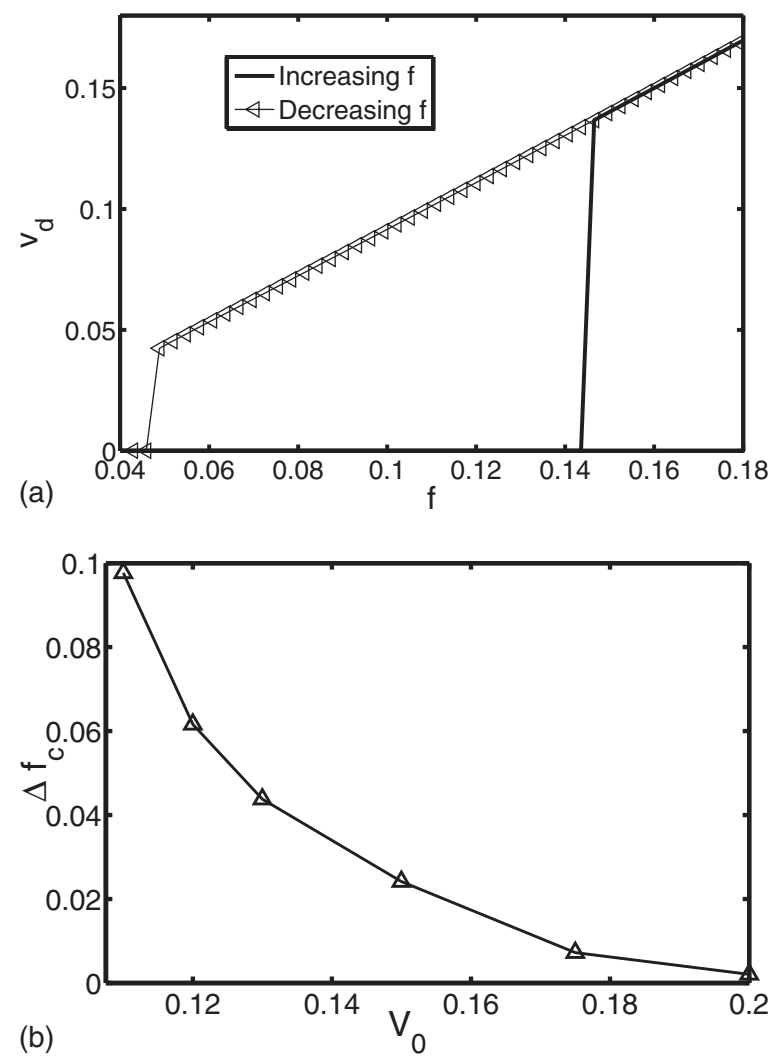

FIG. 2. (a) Discontinuous depinning of the commensurate phase for relatively low pinning strength $\left(\delta_{m}=0.3125, V_{0}=0.11\right)$. (b) Dependence of $\Delta f_{c}$ on $V_{0}$ for $\delta_{m}=0.3125$.

expects that overdamped dynamics should be able to described the hysteresis behavior in two dimensions provided the model incorporates both elastic and plastic deformations. For charge density waves, where the pinning potential is disordered, a field theoretical model has been introduced which allows for dislocations as well as thermal fluctuations [29] through amplitude and phase fluctuations, and shows both elastic and hysteretic behavior in agreement with experiments [30]. In absence of disorder, however, the possible hysteresis behavior in such models has not been investigated.

Recently a phase field crystal (PFC) model was introduced [31-33] that allows for both elastic and plastic deformations in the solid phase. In this formulation a free energy functional is introduced which depends on the field $\psi(\vec{r}, t)$ that corresponds to the particle number density averaged over microscopic times scales. The free energy is minimized when $\psi$ is spatially periodic (i.e., crystalline) in the solid phase and constant in the liquid phase. By incorporating phenomena on atomic length scales the model naturally includes elastic and plastic deformations, multiple crystal orientations, and anisotropic structures in a manner similar to other microscopic approaches such as molecular dynamics. However, the PFC model describes the density on a diffusive and not the real microscopic times scales. It is therefore computationally much more efficient.

In our previous works $[34,35]$ we demonstrated how the influence of an external periodic pinning potential can be incorporated in the PFC model. Such a model provides a continuum description of pinned lattice systems. The pinning 


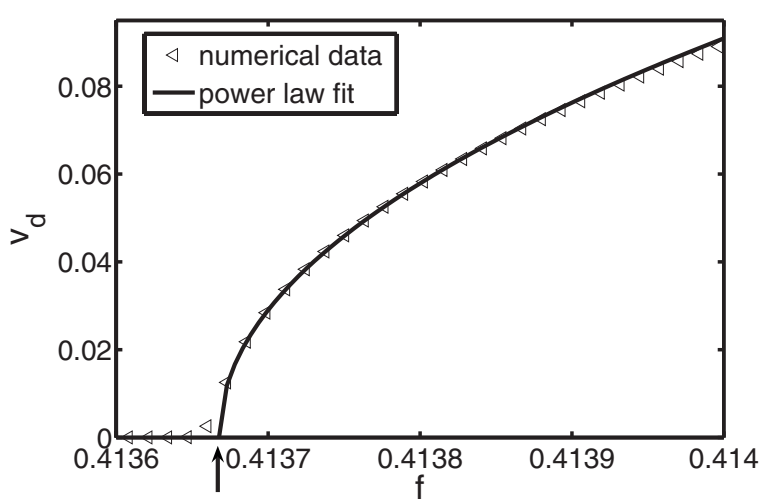

FIG. 3. Continuous depinning transition for relatively high pinning strength $\left(\delta_{m}=0.3125\right.$ and $\left.V_{0}=0.250\right)$. The arrow marks the value of the critical depinning force. The triangles represent the numerical data while the continuous line is a fit to $\left(f-f_{c}\right)^{\zeta}$. The best fit is obtained for the exponent $\zeta=0.50 \pm 0.03$.

potential is chosen such that it allows the occurrence of both $C$ and $I$ phases as ground states of different symmetries in the model. In Ref. [34] part of the phase diagram as function of pinning strength and lattice mismatch between the pinning potential and the PFC was mapped out. Numerical minimization was used to find the minimum free-energy configurations and provide details on the topological defects in the boundary region. In particular, we found that the transition from the $I$ to the $C$ phase remains discontinuous for all values of the mismatch studied in Ref. [34]. We also performed a detailed Voronoi analysis of the defects throughout the transition region. In Ref. [35] the equilibration method was improved and the range of mismatches extended to include both positive and negative mismatches.

In the present work we focus on the case where the PFC under an external periodic potential without disorder introduced in Ref. [34] is driven by an external force in the absence of thermal fluctuations. To this end, we first present improved detailed phase diagrams of the model both in 1D and 2D. The main focus of the present work is on the influence of an external driving force on the pinned $C$ phase, which we study for different values of mismatch and pinning strengths for $1 \mathrm{D}$ and $2 \mathrm{D}$ systems. As expected, due to the competition between the pinning potential and the driving force there is a depinning transition at $f_{c}$ for a finite driving force $f$. We demonstrate that within a certain range of parameters the depinning transitions are continuous, and find that both in $1 \mathrm{D}$ and 2D the corresponding power-law exponent is $\zeta=0.5$ in agreement with the expected value $[13-15,22]$. We also characterize structural changes of the system close to the depinning transition. For large pinning strength transverse depinning transitions in the moving state are also found. Surprisingly, for sufficiently weak pinning potential we find a discontinuous depinning transition with hysteresis even in one dimension although overdamped dynamical equations are used.

\section{THE PHASE FIELD CRYSTAL UNDER A PERIODIC POTENTIAL AND A DRIVING FORCE}

For the phase field crystal in the presence of pinning potential [31-34], the free energy functional can be written in dimensionless form as

$$
F=\int d \vec{x}\left[\frac{\psi}{2}\left[r+\left(1+\nabla^{2}\right)^{2}\right] \psi+\frac{\psi^{4}}{4}+V(\vec{x}) \psi(\vec{x})\right],
$$

where $r$ is a temperature-dependent quantity and $V(\vec{x})$ is an external potential which represents the effect of the substrate. This model can be derived directly from the classical density functional theory [33] of freezing by expanding around the properties of a liquid in coexistence with a solid phase. More specifically it can be shown [36] that $r$ is proportional to the difference between the isothermal compressibility of the liquid and the elastic energy of crystalline phase. Furthermore the length scales in this model have been scaled by the nearest neighbor distance in the coexisting liquid state.

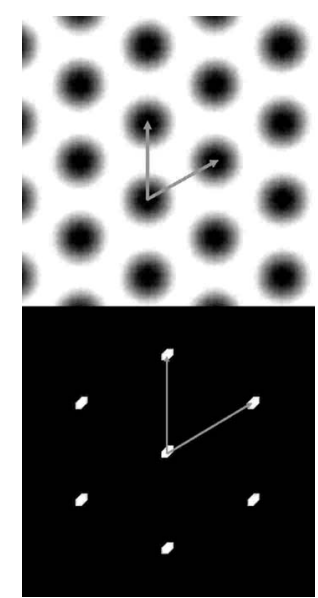

(a)

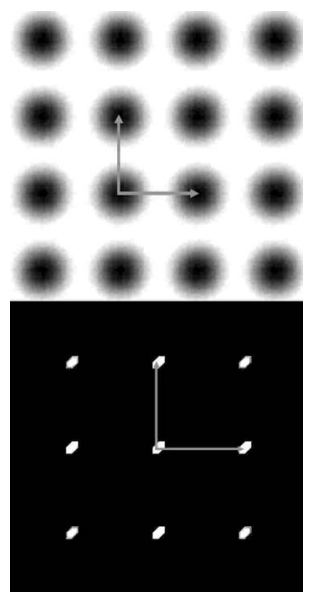

(b)

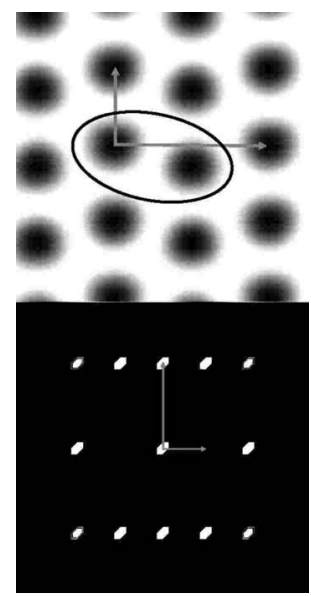

(c)

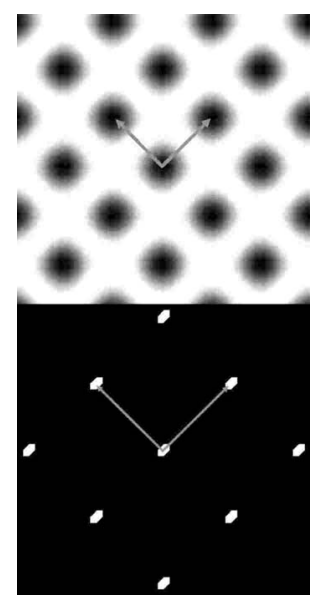

(d)

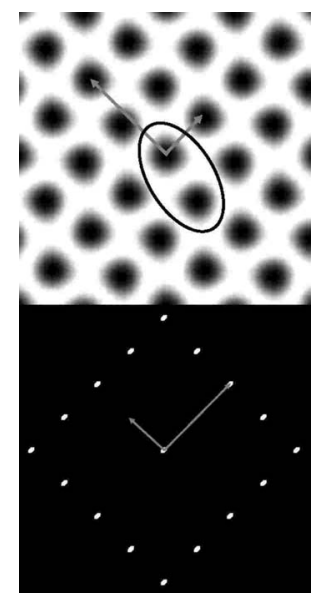

(e)

FIG. 4. The phases that minimize the free-energy, according to Ref. [35]: (a) hexagonal, (b) square $(1 \times 1),(c)$ square $(2 \times 1)$, (d) square $c(2 \times 2)$, and (e) square $2 \sqrt{2} \times \sqrt{2}$. The upper panels represent the density plotted in a gray color map and the corresponding lattice vectors, while the lower panels show the structure factors and the relevant reciprocal lattice vectors. The black contours in Figs. 4(c) and 4(e) show the bases which generate the $(2 \times 1)$ and $(2 \sqrt{2} \times \sqrt{2})$ lattices. 

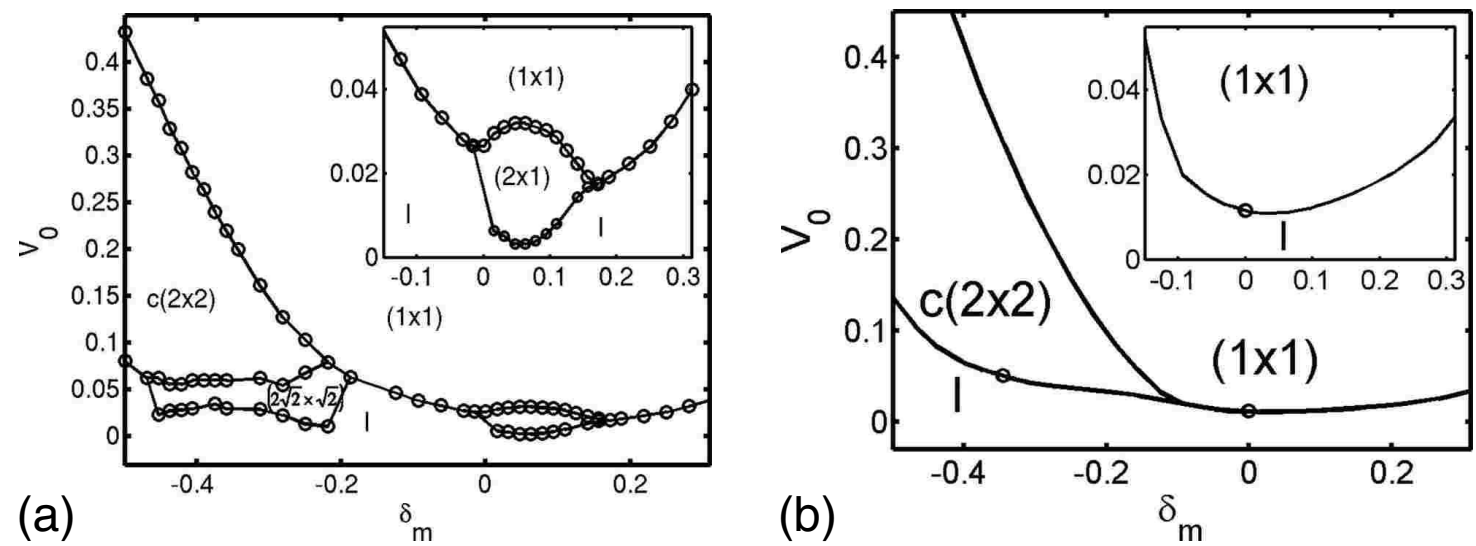

FIG. 5. The phase diagram in terms of the pinning strength $\left(V_{0}\right)$ and mismatch $\delta_{m}$ calculated (a) numerically, according to Ref. [35] and (b) analytically using approximation of the density given by Eq. (9). The insets in (a) and (b) show the phase diagram close to $\delta_{m}=0$. The circles in (b) mark the values for which the approximation for the density given by Eq. (9) breaks down.

In the absence of the pinning potential the equilibrium minimum energy configuration of the system depends on the parameter $r$ and the average density $\bar{\psi}=\frac{1}{V_{d}} \int d \vec{x} \psi(\vec{x})$ [32], where $V_{d}$ is the system volume in $d$ dimensions. In 2D, the solid phase corresponds to a triangular lattice. The length scale chosen here corresponds to $k_{0}^{-1}$, where $k_{0}$ $=2 \pi /\left(a_{t} \sqrt{3} / 2\right)=1$, with $a_{t}$ as the lattice constant of the intrinsic triangular lattice. When the external pinning potential is present, the competition between the length scales associated with the intrinsic ordering and the pinning potential can lead to complicated phases depending on the parameters chosen in the energy functional $[34,35]$. We choose an external pinning potential of a simple periodic form $V=V_{0} \cos \left(k_{s} x\right)$ in $1 \mathrm{D}$, and $V=V_{0}\left[\cos \left(k_{s} x\right)+\cos \left(k_{s} y\right)\right]$ in 2D. The wave vector $k_{s}$ is related to the periodicity of the pinning potential $a_{s}$, such that $k_{s}=2 \pi / a_{s}$.

We define the relative mismatch $\delta_{m}$ between the external potential and the PFC as

$$
\delta_{m}=\left(1-k_{s}\right) .
$$

The response of the system to a driving force can be obtained by including a convective derivative $\vec{f} \cdot \vec{\nabla} \psi$, to the original PFC model [32]. Thus, the dynamical equation of motion for the phase field is given by

$$
\frac{\partial \psi}{\partial \tau}=\nabla^{2} \frac{\delta F}{\delta \psi}+\vec{f} \cdot \vec{\nabla} \psi=\nabla^{2}\left\{\left[r+\left(1+\nabla^{2}\right)^{2}\right] \psi+\psi^{3}+V\right\}+\vec{f} \cdot \vec{\nabla} \psi .
$$

For the forces considered in this work (typically $\vec{f}=f \hat{x}$ ), the convective term does not change the average value of the density field.

In contrast to the usual classical microscopic characterization of particle positions and velocities, the measurement of an average drift velocity in response to an external driving force $\vec{f}$ requires some discussion. In the PFC model the maxima of the density field that define the lattice structure cannot always be interpreted as individual particles, since vacancies may be present in the system. The conservation law in the model concerns the local density field, not the number of maxima in the field. This becomes evident in the driven PFC model, where the motion of the density field close to depinning may be more akin to flow in a continuous medium than the motion of discrete particlelike objects. Thus, defining the drift velocity in terms of the density field maxima is computationally more demanding to implement. We have found that measuring the drift velocity $v_{d}$ from the rate of change of the gradient of the density field gives consistent results, in absence of thermal fluctuations. We have used the following definition:
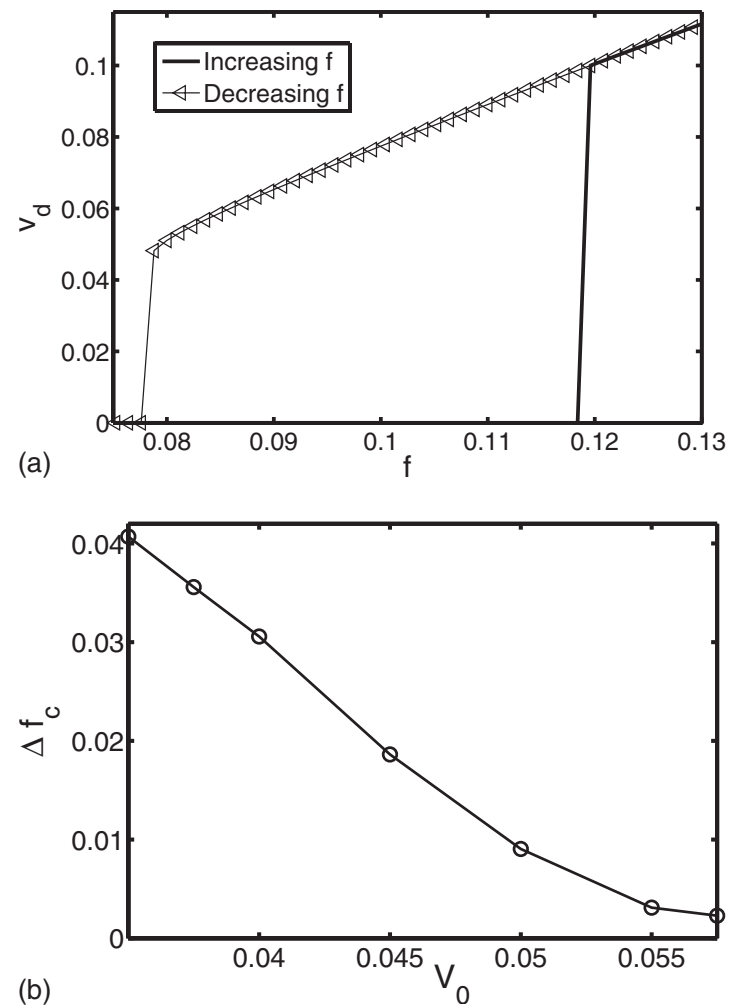

FIG. 6. (a) The variation of the velocity with respect to the external force for a discontinuous transition for the commensurate $(1 \times 1)$ phase $\left(\delta_{m}=0.125, V_{0}=0.0350\right)$ and $(b)$ the variation of the gap $\Delta f_{c}$ vs $V_{0}$ for the same mismatch $\delta_{m}=0.125$. 


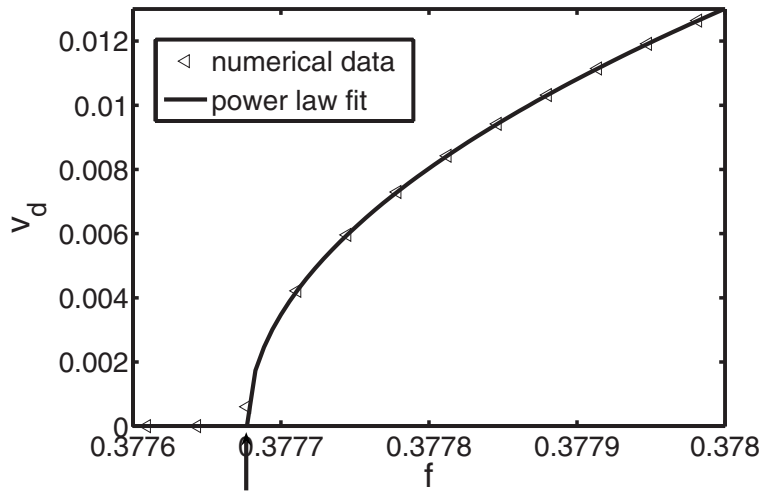

FIG. 7. Dependence of the velocity on the external force for a continuous transition for the $(1 \times 1)$ phase $\left(\delta_{m}=0.125, V_{0}=0.0900\right)$. The vertical arrow marks the critical force $f_{c}$. The triangles represent the numerical data, while the continuous line is a power-law fit with $\zeta=0.50 \pm 0.03$.

$$
\left.v_{d} \equiv\left\langle\langle|\partial \psi / \partial t|\rangle_{\vec{x}} /|\partial \psi / \partial x|\right\rangle_{\vec{x}}\right\rangle_{t},
$$

where the subscripts $\vec{x}$ and $t$ in the brackets denote averaging over space and time, respectively. Alternative definitions were also considered, but this particular form proved the most statistically accurate.

Although the definition of the drift velocity $v_{d}$ according to Eq. (6) can be used to determine the velocity response along the direction of the driving force, it is not particularly useful in the study of the response in the transverse direction since it is not a vector quantity. In order to study the transverse response it is more convenient to determine the average velocity directly from the positions of the local peaks in $\psi(\vec{x})$. This requires locating such peaks as a function of time

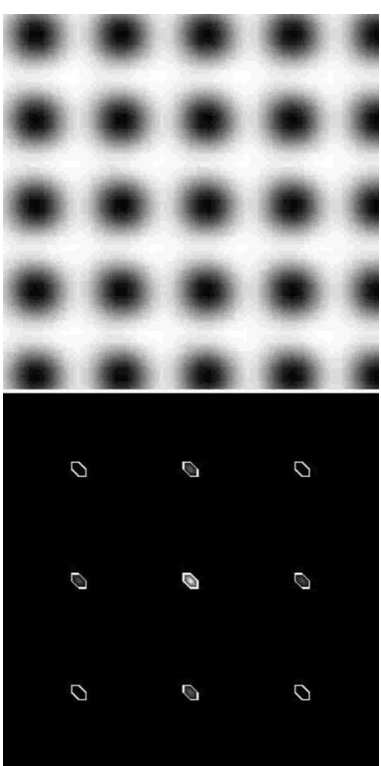

(a)

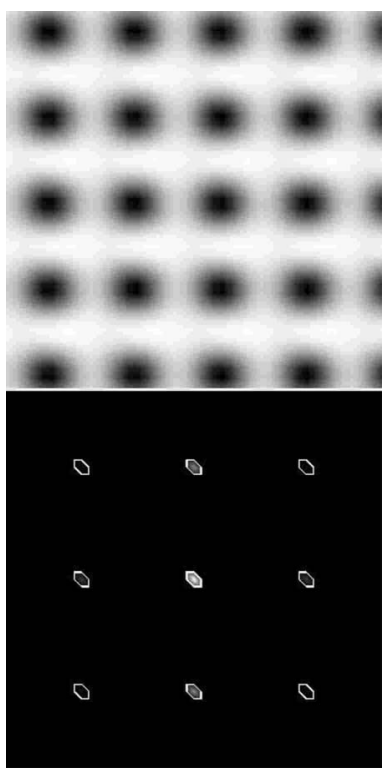

(b)

during the numerical simulation. We have developed a computational method which determines the location and velocity of each individual peak in presence of the external force and thermal fluctuations [37]. The method to locate the peaks is based on a particle location algorithm used in digital image processing [38]. The drift velocity for the lattice of density peaks is obtained from the peak velocities $\vec{v}_{i}$ as

$$
\vec{v}_{P}=\left\langle\frac{1}{N_{P}} \sum_{i=1}^{N_{P}} \vec{v}_{i}(t)\right\rangle_{t},
$$

where $N_{P}$ is the number of peaks. We find that the definitions of the velocity from Eqs. (6) and (7) give consistent results for the longitudinal depinning in absence of thermal fluctuations. However, in presence of thermal fluctuations only the definition from the peak velocities (7) is able to separate the contribution to the drift velocity due to the driving force from thermal noise contributions.

\section{RESULTS}

In this section, we present results obtained for the static and dynamic properties of the PFC model. Numerically, we study the system properties by integrating Eq. (5) using a simple Euler algorithm and the time derivatives are approximated by a forward finite difference with the time step $d t$ $=0.005$ (the time scale corresponds to the diffusion time over the length scale $k_{0}^{-1}$ ). For the $1 \mathrm{D}$ case, the density field was discretized on a uniform grid with $d x=\pi / 4$, while for the 2D case we used a square uniform grid with $d x=d y=\pi / 4$. The Laplacians are evaluated in 1D using a central difference, while for the 2D the "spherical Laplacian" is used [32,39]. In both the equilibrium and driven situations, fully periodic

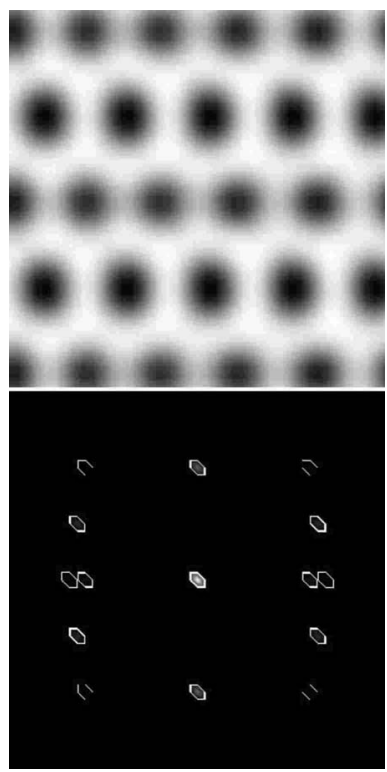

(c)

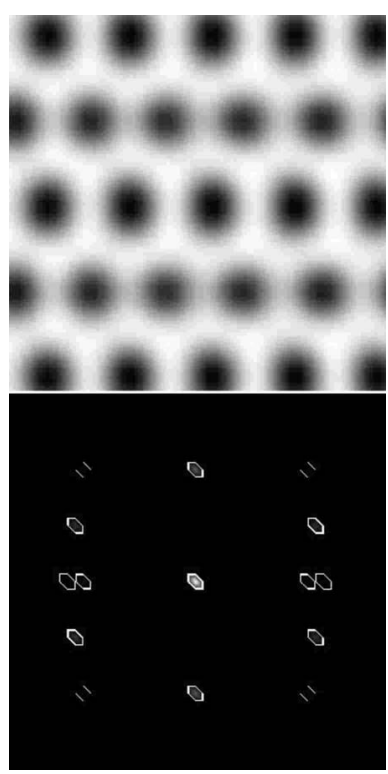

(d)

FIG. 8. Change of the lattice structure (upper panels) and the corresponding structure factor (lower panels) with the applied force for $\delta_{m}=0.125, V_{0}=0.0350$, where depinning is discontinuous. Image (b) corresponds to $f=0.11$ with a nonmoving initial configuration, while for (c) the applied force is the same but the initial configuration is a moving one. The case (a) $f=0.07$, (d) $f=0.13$ are outside of the hysteresis region and same result is obtained with moving or nonmoving initial configuration. 
boundary conditions have been used. Note that for the conserved time-dependent Ginzburg-Landau (TDGL) equation (5), the addition of the driving force of the form $f(\partial \psi / \partial x)$ preserves the local conservation of $\bar{\psi}$ under periodic boundary conditions. For the study of the static properties $\vec{f}$ is set to zero. For a given value of the mismatch, the pinning strength $V_{0}$ is increased from zero to a maximum in steps of $d V_{0}$ and then decreased back to zero. Each time the pinning strength is changed we allow the system to equilibrate. The final state corresponds to a configuration that minimized the energy functional.

For the study of the influence of a driving force we choose the mismatch and pinning strength such that the system is initially in a commensurate state. The driving force $\vec{f}$ is then increased from zero to a maximum value $|\vec{f}|>f_{c}$ and then decreased back to zero. In the case where the depinning transition at $f_{c}$ is continuous, we determine the corresponding depinning exponent $\zeta$ in the limit $|\vec{f}| \rightarrow f_{c}$. Unless specified the force is applied in the $x$ direction, i.e., $\vec{f}=f \hat{x}$.

\section{A. Phase diagram and nonlinear response for a 1D system}

\section{Equilibrium properties}

The static properties of the pinned PFC in 1D $(f=0)$ are presented as a phase diagram in the $V_{0}-\delta_{m}$ plane shown in Fig. 1. For comparison, we include in Fig. 1 the phase boundaries obtained analytically and numerically from Eq. (5). The analytical phase boundary was obtained by minimizing the free energy $F[\psi(x)]$, expanding the density field as

$$
\psi(x)=A_{1} \cos (x)+A_{2} \cos \left(k_{s} x\right)+A_{3} \cos \left[\left(2-k_{s}\right) x\right],
$$

where the last term accounts for the distortion of the lattice.

Next we investigate the influence of an external force on the $1 \mathrm{D}$ commensurate phase. For this purpose, the parameters are chosen such that $r=-1 / 4$ and $\bar{\psi}=0$. Depending on the values of the mismatch and pinning strength different behavior is found when the driving force is added. Several values of mismatch between 0.3125 and -0.50 were investigated. For $\delta_{m} \geq-0.3$ two types of depinning are present, discontinuous and continuous. For values of the pinning strength close to the $I-C$ phase transition, the depinning transition is discontinuous. The dependence of the drift velocity as a function of the driving force exhibits a hysteresis [see Fig. 2(a)]. The gap $\Delta f_{c}=f_{c}^{\text {in }}-f_{c}^{\text {de }}$ decreases when the pinning strength increases [Fig. 2(b)], which indicates that the transition becomes continuous for large enough $V_{0}$.

Note that for $\delta_{m} \lesssim-0.3$ the depinning transition of a $C$ pinned phase is continuous for all values of the pinning strength, while for $\delta_{m} \gtrsim-0.3$ only for values of pinning strength above a certain threshold. In Fig. 3, we show the behavior of a continuous depinning transition for $\delta_{m}$ $=0.3125, V_{0}=0.11$. The dependence of the drift velocity on the force follows a power law $v_{d} \propto\left(f-f_{c}\right)^{\zeta}$, as can be seen in Fig. 3. The exponent $\zeta$ does not depend of the pinning strength and it is equal to $0.50 \pm 0.03$ in all cases studied here. This result can be understood as follows. When the pinning potential is periodic and large in magnitude the neighboring phases are weakly coupled and the system should behave as a single particle in a periodic potential [13-15,22]. This effective single particle behavior which is expected to describe the threshold behavior for a commensurate phase in absence of defects, is independent of the dimension, leading to a depinning exponent [13-15,22] $\zeta$ $=1 / 2$. We also find that the critical force increases with the pinning strength and for $\delta_{m} \gtrsim-0.3$ has linear dependence on the pinning strength, while for $\delta_{m} \lesssim-0.3$ its dependence on pinning becomes sublinear. Finally, we also note that for large driving forces $f \gg f_{c}$ the system is totally depinned and the dependence of the drift velocity on the force follows Eq. (2) with a linear dependence.

\section{B. Phase diagram for a 2D system}

Next we present a summary of the static properties of the 2D PFC model in the presence of the external pinning potential $V(x, y)=V_{0}\left[\cos \left(k_{s} x\right)+\cos \left(k_{s} y\right)\right]$. The parameters chosen are $r=-1 / 4$ and $\bar{\psi}=-1 / 4$. Analytically, we consider the density to be a sum of hexagonal and square modes

$$
\begin{aligned}
\psi(x, y)= & A_{t}\left(\cos (x \sqrt{3} / 2) \cos (y / 2)-\frac{1}{2} \cos (y)\right) \\
& +A_{s 1}\left[\cos \left(k_{s} x\right)+\cos \left(k_{s} y\right)\right] \\
& +A_{s 2} \cos \left(k_{s} x\right) \cos \left(k_{s} y\right) \\
& +A_{c} \cos \left(\frac{k_{s}}{2} x\right) \cos \left(\frac{k_{s}}{2} x\right)+\bar{\psi}
\end{aligned}
$$

For small values of the pinning strength the system is in a hexagonal I phase for all mismatches [Fig. 4(a)]. When the pinning strength is large enough, the system will be in one of the commensurate phases, of which the $(1 \times 1)$ phase is an exact match with the pinning potential here [Fig. 4(b)].

The other ordered phases are higher commensurate phases, which exist only when one of the reciprocal lattice vectors for the commensurate phase is close to the wave vectors of the square pinning potential [35]. One of these phases is the $c(2 \times 2)$ phase [Fig. $4(d)]$ in which every second site of the lattice of the pinning potential corresponds to the maximum in the phase field [1]. This state is favored for mismatch values close to $1-\sqrt{2}$. Another higher commensurate phase is the $(2 \times 1)$ [Fig. $4(\mathrm{c})$ ] which is generated by a translation of the basis with the reciprocal lattice vectors of the a $c(2 \times 1)$ lattice [1]. Finally, the $(2 \sqrt{2} \times \sqrt{2})$ phase [Fig. $4(\mathrm{e})]$ is similar to the $(2 \times 1)$ phase. The lattice is generated by a translation of the basis with vectors which are rotated $45^{\circ}$ with respect to the pinning potential and the magnitudes of the vectors are $2 \sqrt{2} a_{s}$ and $\sqrt{2} a_{s}$. The phase is favored for mismatch values close to -0.27 .

The transitions between the different phases are found by investigating the positions and the heights of the peaks in the structure factor. The results of our extensive numerical calculations are summarized in the phase diagram of Fig. 5(a) which has been taken from Ref. [35].

\section{Nonlinear response of the $(1 \times 1)$ phase}

We now turn to the influence of an external driving force for the pinned, commensurate $(1 \times 1)$ and $c(2 \times 2)$ phases. 


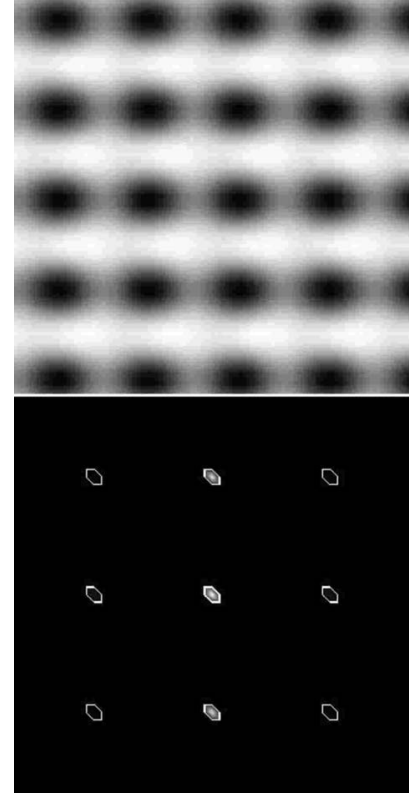

(a)

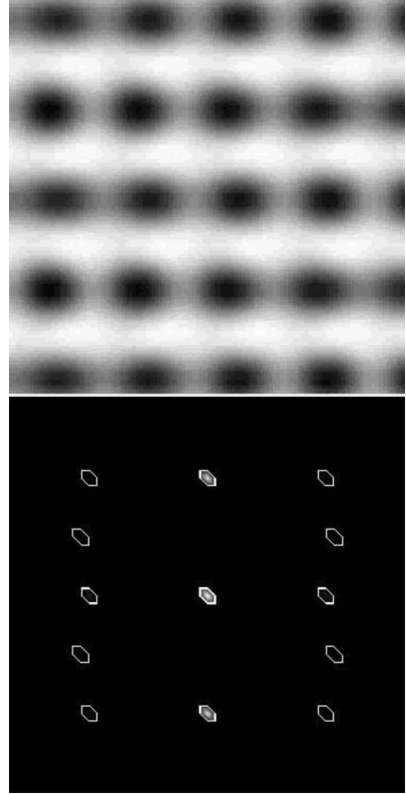

(b)

FIG. 9. Change of the structure factor with the applied force for $\delta_{m}=0.125, V_{0}=0.0900$, where depinning is continuous. The images correspond to (a) $f=0.3776$ [before the depinning transition marked by the vertical arrow in Fig. 7(a)], (b) $f=0.3782$ (after the depinning transition).

Depending on the values of $\delta_{m}$ and $V_{0}$, for both phases we find both discontinuous and continuous depinning transitions. For $\delta_{m} \geqslant-0.2$ and $V_{0} \geqslant 0.09$ both continuous and discontinuous depinning mechanisms were found for the commensurate $(1 \times 1)$ phase. For smaller values of the pinning strength close to the IC transition, only discontinuous depinning transitions were found. Similar to the 1D case, we identify two values of the critical forces for a discontinuous depinning, namely, $f_{c}^{\text {in }}$ for when the force is increased and $f_{c}^{\mathrm{de}}$ when the force is decreased back to zero. Figure 6(a) shows the velocity dependence with respect to the applied force for a discontinuous transition. We have also tested the effect of thermal fluctuations here and find that for temperatures low enough the hysteresis remains unchanged up to some value which depends on the mismatch and pinning strength. The gap $\Delta f_{c}=f_{c}^{\text {in }}-f_{c}^{\text {de }}$ for a given $\delta_{m}$ decreases as the pinning strength is increased [Fig. 6(b)]. Finally, when the gap $\Delta f_{c}$ vanishes the depinning transition becomes continuous. In this regime we find that the sliding velocity follows a power low $v_{d} \propto\left(f-f_{c}\right)^{\zeta}$ (Fig. 7), consistent with $\zeta=0.5$ in all cases studied here as in the $1 \mathrm{D}$ case. We note that for $\delta_{m} \leqslant-0.2$ only continuous depinning transitions were found for all values of the pinning strength.

Both depinning mechanisms are accompanied by structural changes. The system changes from a commensurate $(1 \times 1)$ phase (below critical threshold) to a distorted hexagonal phase (Figs. 8 and 9).

\section{Nonlinear response of the $c(2 \times 2)$ phase}

Similar to the $(1 \times 1)$ phase, the commensurate $c(2 \times 2)$ phase also exhibits both discontinuous and continuous depin-
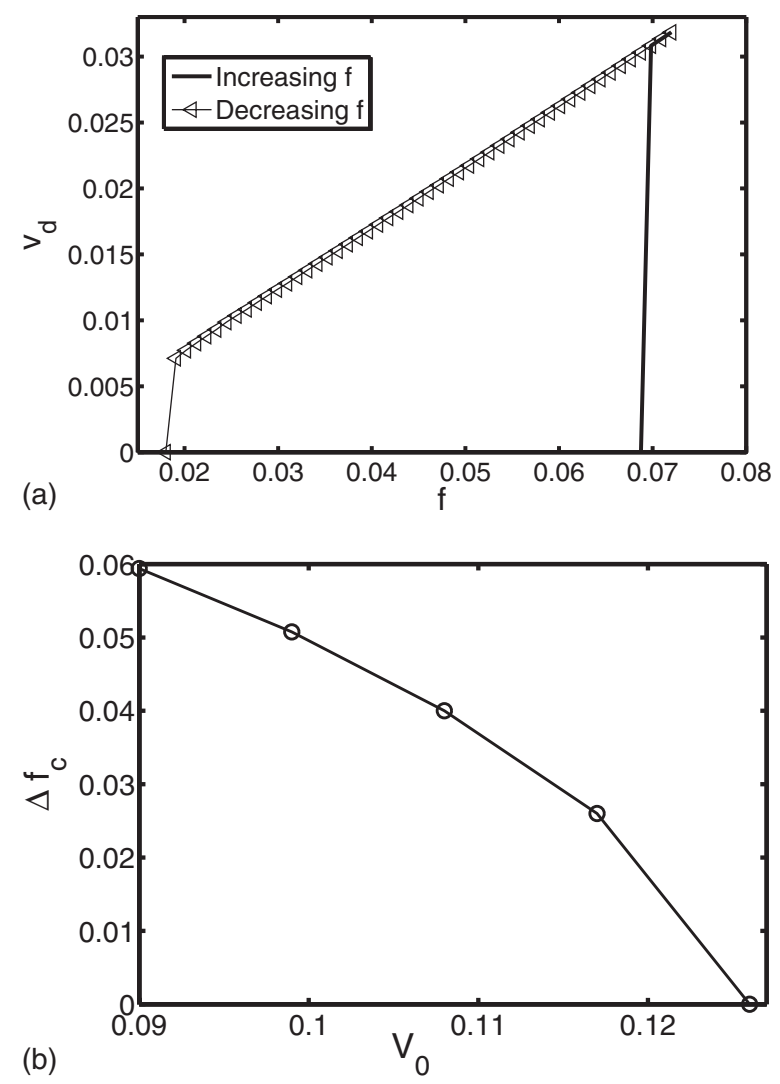

FIG. 10. (a) The variation of the velocity with respect to the external force for a discontinuous transition for the $c(2 \times 2)$ phase $\left(\delta_{m}=-0.50, V_{0}=0.099\right)$ and (b) $\Delta f_{c}$ vs $V_{0}$ for $\delta_{m}=-0.50$.

ning. For low value of pinning strength discontinuous depinning transition was found (Fig. 10), while for large values of the pinning strength the depinning becomes continuous (Fig. 11) with the exponent $\zeta=0.5$. In both cases the structure of the systems changes when the force is applied from a $C$ phase to a distorted hexagonal depinned phase (Figs. 12 and 13).

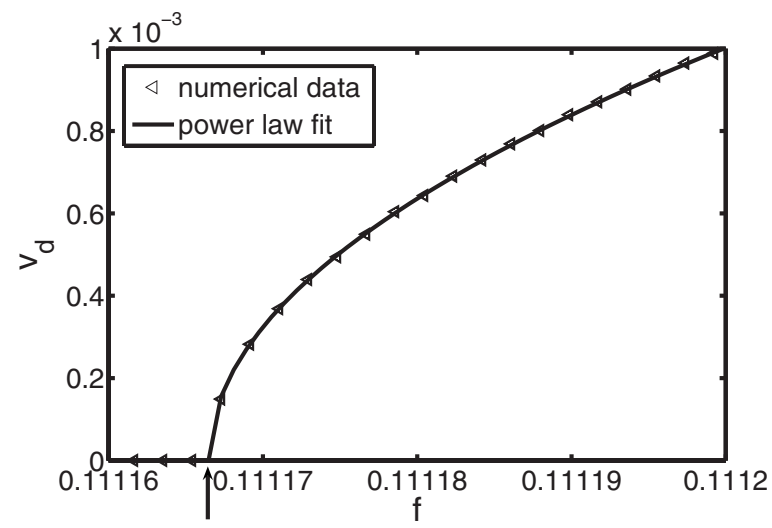

FIG. 11. The variation of the velocity with respect to the external force for a continuous transition $\left(\delta_{m}=-0.50, V_{0}=0.207\right)$ for the $c(2 \times 2)$ and the corresponding power law fit. The vertical arrow marks the value of the critical depinning force. The triangles represent the numerical data, while the continuous line the power law fit with $\zeta=0.50 \pm 0.03$. 


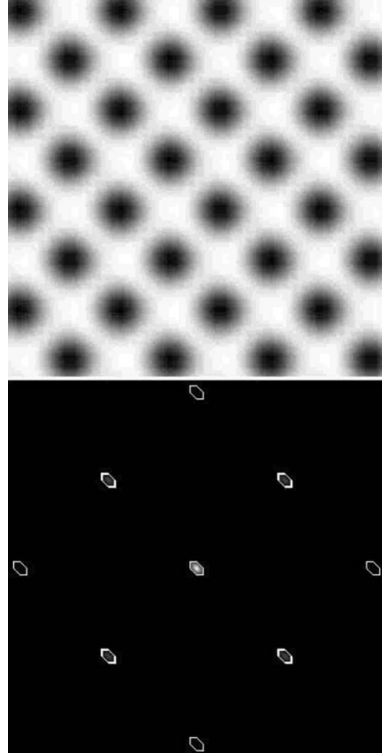

(a)

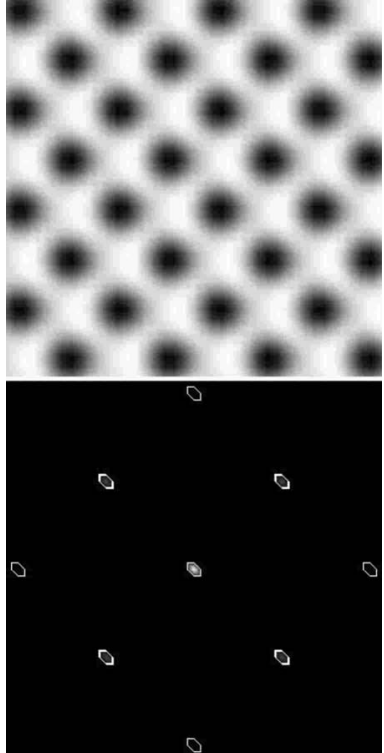

(b)

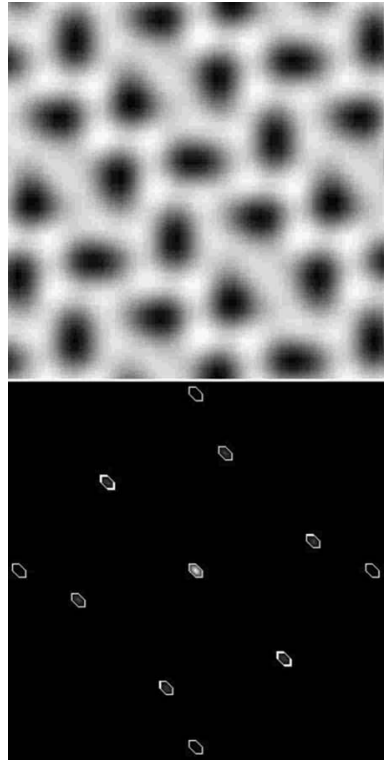

(c)

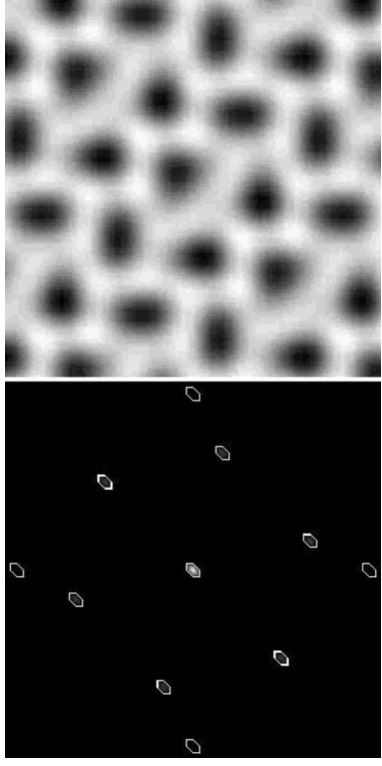

(d)

FIG. 12. Change of the lattice structure (upper panels) and the corresponding structure factor (lower panels) with the applied force for $\delta_{m}=-0.5, V_{0}=0.099$ for $c(2 \times 2)$, where depinning is discontinuous. Image (b) corresponds to $f=0.10504$ with a nonmoving initial configuration, while for (c) the applied force is the same but the initial configuration is a moving one. The cases (a) $f=0.018$, (d) $f=0.13$ are outside of the hysteresis region and same result is obtained with moving or nonmoving initial configuration.

While for the cases presented here the structural changes occur when the system starts to depin, it is also possible for changes to occur for forces below the critical threshold. For some values of the mismatch and pinning strength, if the force is rotated $45^{\circ}$ the $c(2 \times 2)$ phase will change first to a $(1 \times 1)$ phase from which the systems depins continuously as described before. Other force induced transitions between commensurate phases with no sliding are also possible.

The hysteresis behavior of the depinning transition found for sufficiently small pinning strength $V_{0}$ and the critical exponent $\xi$ for the continuous transition for larger $V_{\text {, }}$ are confirmed by calculations of peak velocity $v_{p}$ from Eq. (7). For large $V_{0}$ where there is no hysteresis, the behavior of $v_{p}$ as function of $f$ show a depinnning transition at a critical force $f_{c}$. A power-law fit of the velocity near $f_{c}$ gives an exponent $\zeta=0.52 \pm 0.03$ which is consistent with the estimate using the velocity definition in Eq. (6).

The determination of the velocity response from the peak positions allows us also to study the response to an additional force $f_{y}$ applied perpendicular to the longitudinal force $f_{x}$ in the moving state. For $f_{x} \gg f_{c}$ the longitudinal velocity $v_{x}$ is proportional to the force since in the moving state the external pinning potential in the direction of the force appears as a time dependent perturbation in a reference system comoving with the lattice, with a vanishing time average [40]. However, the pinning potential remains static in the transverse direction [40]. One then expects that for sufficiently larger pinning strength, a transverse depinning transition is possible for increasing force $f_{y}$ while $f_{x}$ is kept fixed. Figure 14(a) shows the behavior of the transverse velocity component $v_{y}$ when an increasing $f_{y}$ is applied in the moving state with fixed $f_{x}>f_{c}$. The transverse critical force $f_{y c}$ decreases with the longitudinal force $f_{x}$ and appears to vanishes at the longitudinal depinning transition $f_{c}$, as shown in Fig. 14(b).

\section{DISCUSSION AND CONCLUSIONS}

In this work we have considered the recently developed phase field crystal model [32] in the presence of an external periodic pinning potential $[34,35]$ and a driving force. As the model naturally incorporates both elastic and plastic defor-

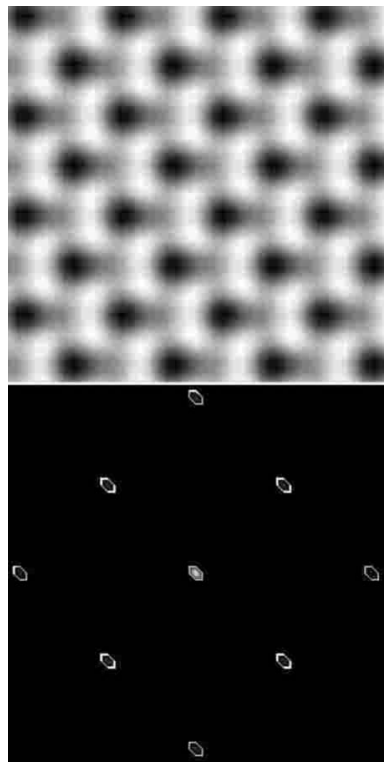

(a)

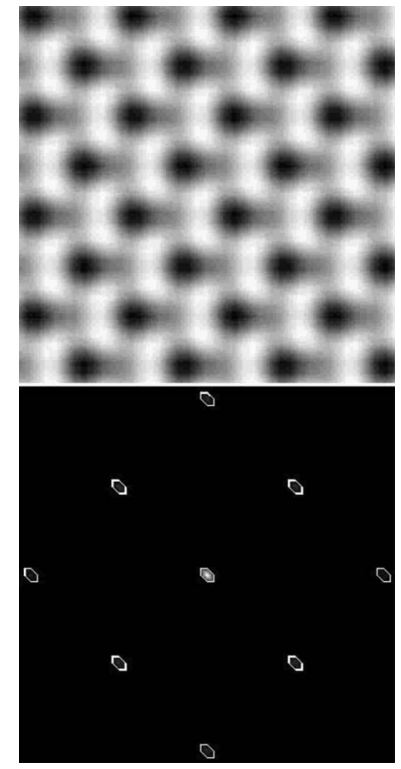

(b)
FIG. 13. Change of the structure factor with the applied force for $\delta_{m}=-0.50, V_{0}=0.207$ for the $c(2 \times 2)$, where depinning is continuous. The images correspond to (a) $f=0.1111664528$ [right before the depinning transition indicated by the vertical arrow in Figs. 11(a) and 11(b)] $f=0.1111672800$ (right after the depinning transition). 

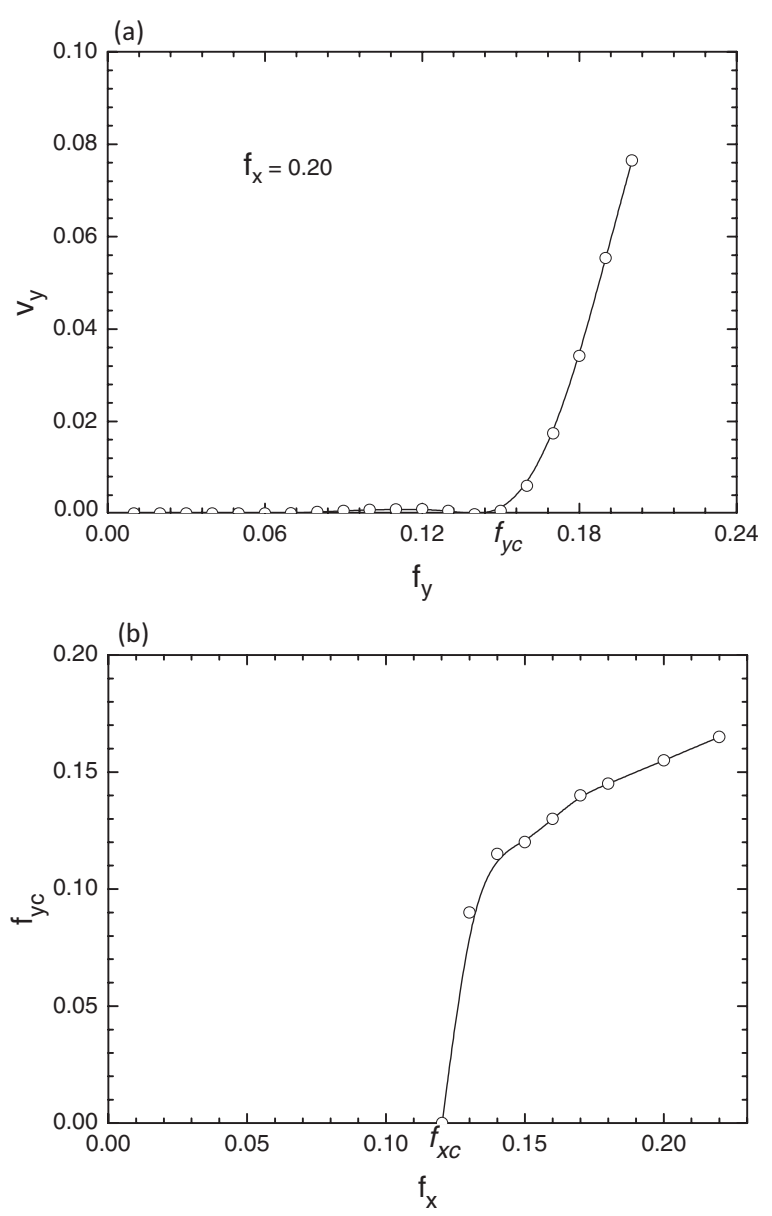

FIG. 14. (a) $v_{y}$ as a function of an additional force $f_{y}$ in the transverse direction, with $f_{x}$ fixed. (b) Critical transverse force $f_{c y}$ as a function of the longitudinal force $f_{x}$. Results for $V_{0}=0.275$, $\delta_{m}=-0.5$.

mations, it provides a continuum description of lattice systems such as adsorbed atomic layers on surfaces or 2D vortex lattices in superconducting thin films, while still retaining the discrete lattice symmetry of the solid phase. The main advantage of the model as compared to traditional approaches is that despite retaining spatial resolution at its lowest length scale its temporal evolution naturally follows diffusive time scales. Thus the numerical simulation studies of the dynamics of the systems can be achieved over realistic time scales, which, for example, in the case of adsorbed atomic systems may correspond to many orders of magnitude over the time scale used in microscopic atomic models. In this work we have exploited this method to determine the phase diagram in one and two dimensions as a function of lattice mismatch, pinning strength, and a driving force.

We have concentrated on the nonlinear response to an external driving force on the most common stable commensurate states, namely, the $(1 \times 1)$ and the $c(2 \times 2)$ phases. These are particularly interesting cases which are relevant for physical systems of current interest and accessible experimentally such as, driven adsorbed layers [9,26], which determine the sliding friction behavior between two surfaces with a lubricant [10] and between adsorbed layers and an oscillating substrate $[11,12]$.
Our results for the phase field crystal model with overdamped dynamics indicate both discontinuous and continuous transitions depending on the magnitude of the pinning strength. For high enough pinning strengths continuous transitions occurred with the velocity near the transition scaling as $\left(f-f_{c}\right)^{1 / 2}$, independent of the dimension of the system. This is as expected, since for a commensurate state in a strong periodic pinning potential, each "particle" acts independently and the model reduces to an effective single particle in a periodic potential, with a known depinning exponent of $1 / 2$. Perhaps more interesting is the observation of discrete transitions and hysteresis loops found at low pinning strengths. In the two-dimensional case, the observed hysteresis behavior is consistent with the arguments and atomistic molecular dynamics simulations of driven adsorbed layers $[9,26]$ indicating that hysteresis remains in the overdamped limit. However, our results show that it disappears for large enough pinning strength. For the discontinuous transition, there are two different critical values $f_{c}^{\mathrm{in}}>f_{c}^{\mathrm{de}}$, correspond to the static and kinetic critical forces, respectively, which lead the to stick and slip motion at low sliding velocities as observed experimentally [10]. The general features observed of hysteresis and power-law behavior near the continuous depinning transition are also of interest for driven charge density waves $[15,29]$ and driven flux lattices $[41,43]$ although in these cases there are important additional effects due to a large degree of disorder in the pinning potential. Whether the present phase field model also shows the same main features observed for the atomistic model in presence of thermal fluctuations [26-28] is an interesting question which will require further investigation.

In addition to the longitudinal depinning transition where the lattice system is moving in the same direction as the driving force, a driven two-dimensional lattice on a periodic potential can also show an interesting behavior for the transverse response in the moving state. When the lattice is already moving along some symmetry direction of the pinning potential the response to an additional force applied in the direction perpendicular to the longitudinal driving force may lead to a depinning transition for increasing transverse force [40]. Such transverse depinning has been found in different driven lattice systems with periodic pinning including driven vortex lattices [41,42] and adsorbed layers [27] in standard molecular dynamics simulations. In the present PFC model, we have obtained similar results for the transverse depinning. Experimentally, some evidence of transverse pinning has been observed in measurements on charge-density waves [44]. Wigner solids [45], and vortex lattices [46], although in these cases disorder in the pinning potential plays a more important role.

While the hysteresis behavior is expected in the presence of inertial terms both in $1 \mathrm{D}$ and $2 \mathrm{D}$, it is quite interesting to see it in 1D when the dynamics being used is overdamped and purely relaxational. In $2 \mathrm{D}$, it can be argued that topological defects such as dislocations can lead to this behavior even with overdamped dynamics but these defects are not available in 1D. It is interesting to speculate that the hysteresis behavior is intimately related to the need for plastic deformations to mediate the transition from one lattice structure to another. Work on these problems is already in progress. 


\section{ACKNOWLEDGMENTS}

This work was supported by joint funding under EU Grant No. STRP 016447 MagDot and NSF DMR Grant No. 0502737 (C.V.A. and T.A-N.). Computations were performed in the CSC's computing environment. CSC is the Finnish IT Center for Science and is owned by the Ministry of Education. E.G. was supported by Fundação de Amparo à Pesquisa do Estado de São Paulo-FAPESP (Grant No. 07/ 08492-9). K.R.E. acknowledges the support from NSF Grant No. DMR-0413062. M.K. has been supported by the Natural Sciences and Engineering Research Council of Canada (NSERC) and SharcNet (www.sharcnet.ca).
[1] M. Schick, Prog. Surf. Sci. 11, 245 (1981).

[2] P. Bak, Rep. Prog. Phys. 45, 587 (1982).

[3] P. F. Tua and J. Ruvalds, Phys. Rev. B 32, 4660 (1985).

[4] E. Fawcett, Rev. Mod. Phys. 60, 209 (1988).

[5] R. M. Fleming, L. F. Schneemeyer, D. E. Moncton, Phys. Rev. B 31, 899 (1985).

[6] J. I. Martín, M. Vélez, J. Nogués, and Ivan K. Schuller, Phys. Rev. Lett. 79, 1929 (1997).

[7] A. Thomy, X. Duval, and J. Regnier, Surf. Sci. Rep. 1, 1 (1980).

[8] R. Pandit, M. Schick, and M. Wortis, Phys. Rev. B 26, 5112 (1982).

[9] B. N. J. Persson, Sliding Friction: Physical Principles and Applications (Springer, Heidelberg, 1998); in Physics of Sliding Friction, edited by B. N. J. Persson and E. Tosatti (Kluwer, Dordrecht, 1996).

[10] H. Yoshizawa, P. McGuiggan, and J. Israelachvili, Science 259, 1305 (1993); 14, 109 (1992); A. D. Berman, W. A. Ducker, and J. N. Israelachvili, in Physics of Sliding Friction, edited by B. N. J. Persson and E. Tosatti (Kluwer, Dordrecht, 1996); M. Urbakh, J. Klafter, D. Gourdon, and J. Israelachvili, Nature (London) 430, 525 (2004).

[11] J. Krim, D. H. Solina, and R. Chiarello, Phys. Rev. Lett. 66, 181 (1991).

[12] A. Carlin, L. Bruschi, M. Ferrari, and G. Mistura, Phys. Rev. B 68, 045420 (2003).

[13] D. S. Fisher, Phys. Rev. Lett. 50, 1486 (1983).

[14] D. S. Fisher, Phys. Rev. B 31, 1396 (1985).

[15] G. Grüner, A. Zawadowski, and P. M. Chaikin, Phys. Rev. Lett. 46, 511 (1981).

[16] S. Tomić, N. Biškup, M. Pinterić, J. U. von Schütz, H. Schmitt, and R. Moret, Europhys. Lett. 38, 219 (1997).

[17] U. Welp, X. L. Xiao, V. Novosad, and V. K. Vlasko-Vlasov, Phys. Rev. B 71, 014505 (2005).

[18] T. C. Wu, J. C. Wang, L. Horng, J. C. Wu, and T. J. Yang, J. Appl. Phys. 97, 10B102 (2005).

[19] J. E. Villegas, M. I. Montero, C.-P. Li, and I. K. Schuller, Phys. Rev. Lett. 97, 027002 (2006).

[20] A. A. Middleton and D. S. Fisher, Phys. Rev. Lett. 66, 92 (1991).

[21] A. A. Middleton, O. Biham, P. B. Littlewood, and P. Sibani, Phys. Rev. Lett. 68, 1586 (1992).

[22] C. R. Myers and J. P. Sethna, Phys. Rev. B 47, 11171 (1993).

[23] T. A. Kontorova and Y. I. Frenkel, Zh. Eksp. Teor. Fiz. 8, 89
(1939).

[24] P. M. Chaikin and T. C. Lubensky, Principles of Condensed Matter Physics (Cambridge University Press, New York, 1995).

[25] E. Granato and S. C. Ying, Phys. Rev. B 59, 5154 (1999).

[26] B. N. J. Persson, Phys. Rev. Lett. 71, 1212 (1993).

[27] E. Granato and S. C. Ying, Phys. Rev. Lett. 85, 5368 (2000); Phys. Rev. B 69, 125403 (2004).

[28] B. N. J. Persson, J. Chem. Phys. 103, 3849 (1995).

[29] M. Karttunen, M. Haataja, K. R. Elder, and M. Grant, Phys. Rev. Lett. 83, 3518 (1999).

[30] K. L. Ringland, A. C. Finnefrock, Y. Li, J. D. Brock, S. G. Lemay, and R. E. Thorne, Phys. Rev. Lett. 82, 1923 (1999).

[31] K. R. Elder, M. Katakowski, M. Haataja, and M. Grant, Phys. Rev. Lett. 88, 245701 (2002).

[32] K. R. Elder and M. Grant, Phys. Rev. E 70, 051605 (2004).

[33] K. R. Elder, N. Provatas, J. Berry, P. Stefanovic, and M. Grant, Phys. Rev. B 75, 064107 (2007).

[34] C. V. Achim, M. Karttunen, K. R. Elder, E. Granato, T. AlaNissila, and S. C. Ying, Phys. Rev. E 74, 021104 (2006).

[35] C. V. Achim, M. Karttunen, K. R. Elder, E. Granato, T. AlaNissila, and S. C. Ying, J. Phys.: Conf. Ser. 100, 072001 (2008).

[36] Z.-F. Huang and K. R. Elder, Phys. Rev. Lett. 101, 158701 (2008).

[37] J. A. P. Ramos, E. Granato, C. V. Achim, M. Karttunen, S. C. Ying, K. R. Elder, and T. Ala-Nissila (unpublished).

[38] J. C. Crocker and D. G. Grier, J. Colloid Interface Sci. 179, 298 (1996).

[39] M. Patra and M. Karttunen, Numer. Methods Partial Differ. Equ. 22, 936 (2006).

[40] P. Le Doussal and T. Giamarchi, Phys. Rev. B 57, 11356 (1998).

[41] C. Reichhardt and F. Nori, Phys. Rev. Lett. 82, 414 (1999).

[42] C. Reichhardt and C. J. Olson Reichhardt, Phys. Rev. Lett. 100, 167002 (2008).

[43] G. Blatter, M. V. Feigel'man, V. B. Geshkeibein, A. I. Larkin, and V. M. Vinokur, Rev. Mod. Phys. 66, 1125 (1994).

[44] N. Markovic, M. A. H. Dohmen, and H. S. J. van der Zant, Phys. Rev. Lett. 84, 534 (2000).

[45] F. Perruchot, F. I. B. Williams, C. J. Mellor, R. Gaal, B. Sas, and M. Henini, Physica B 284, 1984 (2000).

[46] J. Lefebvre, M. Hilke, and Z. Altounian, Phys. Rev. B 78, 134506 (2008). 